\title{
Diamagnetic Behavior in Nanoparticle Hematite?
}

\author{
Petar A. Petrov' ${ }^{1}$, Arfan Ali' ${ }^{2}$, David K. Potter ${ }^{1}$ \\ ${ }^{1}$ Department of Physics, University of Alberta, Edmonton, Canada \\ ${ }^{2}$ Shell UK Limited, Aberdeen, UK \\ Email: dkpotter@ualberta.ca
}

How to cite this paper: Petrov, P.A., Ali, A. and Potter, D.K. (2017) Diamagnetic Behavior in Nanoparticle Hematite? Journal of Modern Physics, 8, 1013-1019. https://doi.org/10.4236/jmp.2017.87063

Received: April 24, 2017

Accepted: May 30, 2017

Published: June 2, 2017

Copyright (C) 2017 by authors and Scientific Research Publishing Inc. This work is licensed under the Creative Commons Attribution International License (CC BY 4.0).

http://creativecommons.org/licenses/by/4.0/

\begin{abstract}
A paper by Mi et al. [1] suggested that certain nano-sized hematite $\left(\alpha-\mathrm{Fe}_{2} \mathrm{O}_{3}\right)$ particles had diamagnetic properties at room temperature. Since diamagnetic behavior is not a property normally attributed to hematite particles (hematite is generally regarded as a canted antiferromagnetic material at room temperature) we decided to test the validity of the suggestions in [1] by performing magnetic susceptibility and magnetic hysteresis measurements on a series of hematite nanoparticles with average sizes of $8 \mathrm{~nm}, 30 \mathrm{~nm}$ and $40 \mathrm{~nm}$ in diameter. We initially considered two possible explanations for the apparent diamagnetic behavior of the nanoparticles in [1]: either 1) the hematite nanoparticles themselves exhibited this unusual diamagnetic behavior, or 2) the diamagnetic response was simply the signal created by a diamagnetic dispersant that was overriding a weak positive magnetic susceptibility signal of the hematite nanoparticles. Our experiments strongly suggested the latter explanation that the apparent "diamagnetic" behavior seen in [1] was caused by a diamagnetic dispersant dominating the magnetic properties of the dispersed hematite nanoparticles.
\end{abstract}

\section{Keywords}

Diamagnetic, Hematite, Nanoparticle, Magnetic Hysteresis, Magnetic Susceptibility

\section{Introduction}

Hematite has a complex magnetic behavior that is highly dependent on temperature and particle size. Hematite has a Néel temperature of about $948 \mathrm{~K}$ [2], and also undergoes a low temperature transition just below room temperature called the Morin transition. Reference [3] found that the Morin transition was highly dependent upon particle size, varying between $241 \mathrm{~K}$ and $256 \mathrm{~K}$ for submicron 
synthetic hematites with particle sizes between $120 \mathrm{~nm}$ and $520 \mathrm{~nm}$. Below its Morin temperature hematite is antiferromagnetic [2]. Above its Morin temperature and below its Néel temperature, hematite behaves as a canted antiferromagnetic material, while above its Néel temperature hematite is paramagnetic [2].

Reference [1] reported diamagnetic hysteresis curve behavior at room temperature in spindle-type hematite nanoparticles $200 \mathrm{~nm}$ long and $80 \mathrm{~nm}$ wide. This result was very unusual considering the normally accepted properties of hematite summarized above. We speculated on two possible reasons for the observed diamagnetic behavior of the hematite nanoparticles in [1]: either 1) the particles did exhibit some unusual diamagnetic behavior for certain sizes or shapes of nanoparticles (perhaps due to their proximity to the stable single domain/superparamagnetic boundary or due to their "spindle" shape), or 2) the diamagnetic magnetic hysteresis behavior was caused by a diamagnetic particle dispersant overwhelming the weak canted antiferromagnetic hematite signal of the nanoparticles at room temperature. The latter explanation seemed much more plausible. We also noticed that the magnetic hysteresis curve for the "diamagnetic" hematite nanoparticle sample in [1] is not a straight line with negative slope and therefore is not a pure diamagnetic signal. Instead the curve shows a distinct change of slope at low applied field strengths. We speculated whether this change of slope might be related to the weak hematite nanoparticle signal. The purpose of our paper was to test some other hematite nanoparticles to see whether we also observe diamagnetic behavior. We firstly measured the magnetic properties of some bulk samples of hematite nanoparticles. Subsequently, in order to test our hypothesis that a diamagnetic dispersant could be responsible for the results in [1], we undertook magnetic measurements on a suitable source of hematite nanoparticles that were very dilutely dispersed in diamagnetic water.

\section{Samples and Methods}

A source of nominally spherical $30 \mathrm{~nm}$ hematite nanoparticles, synthesized using the SOL-GEL method, was purchased from the MTI corporation. X-ray diffraction (XRD) and transmission electron microscopy (TEM) were used to confirm the chemical composition and average particle size of the nanoparticles. In order to prepare the $30 \mathrm{~nm}$ nanoparticles for the TEM imaging a small amount of the hematite nanopowder was immersed in ethanol solution and then sonicated for 40 minutes in order to increase particle dispersion. A drop of the solution was then placed on an observation plate and left to air dry. The observation plate was then inserted into the TEM and photomicrographs were taken of the particles in the sample.

A bulk volume magnetic susceptibility measurement of the nanoparticles at room temperature was conducted using a Bartington MS2E probe sensor connected to an MS2 meter. A measurement was taken directly from a large sample of the nanopowder in order to obtain a bulk volume magnetic susceptibility val- 
ue of the hematite nanoparticles alone without being dispersed in any kind of matrix. A more accurate independent measurement of the volume magnetic susceptibility at room temperature was also made using a Bartington MS2B magnetic sensor connected to an MS2 meter. A known amount of the nanoparticle powder was placed in the $10 \mathrm{ml}$ sample holder and the volume magnetic susceptibility was measured. The mass of the sample was also determined and used to calculate the mass magnetic susceptibility.

Subsequently, a variable field translation balance (VFTB) was used to obtain the magnetic hysteresis loop of the $30 \mathrm{~nm}$ hematite nanoparticles at room temperature. The hematite nanoparticles in this case were mixed with diamagnetic calcium fluoride $\left(\mathrm{Ca}_{2} \mathrm{~F}\right)$ powder to increase particle dispersion. The concentration was 1 part hematite nanoparticle powder to 8 parts calcium fluoride by mass. The magnetic hysteresis loop of the mixture was then measured using the VFTB up to a maximum applied field value of 1 Tesla $(1000 \mathrm{mT})$.

In order to test suitable samples of very dilutely dispersed hematite nanoparticles in a diamagnetic matrix, we obtained some hematite nanoparticle samples dispersed in diamagnetic water from Particular GmbH. We tested two samples, one containing hematite nanoparticles of $8 \mathrm{~nm}$ and the other of $40 \mathrm{~nm}$. The hematite nanoparticles were synthesised by laser ablation in water, and their sizes were accurately determined by a Malvern laser particle size analyser. The concentration in water of the $8 \mathrm{~nm}$ nanoparticles was $140 \mathrm{mg} / \mathrm{L}$ and that of the 40 $\mathrm{nm}$ nanoparticles was $160 \mathrm{mg} / \mathrm{L}$. A VFTB was used to obtain the magnetic hysteresis loops of each hematite nanoparticle solution. For comparison a further magnetic hysteresis loop was taken of a sample of the water used for the dispersion.

\section{Results and Discussion}

The chemical composition of the $30 \mathrm{~nm}$ hematite nanoparticles was confirmed by the XRD analysis (Figure 1). The observed diffraction peaks coincided with those expected for hematite (indicated by the red vertical lines in Figure 1). TEM photomicrographs of the hematite nanoparticles confirmed an average particle size of approximately $30 \mathrm{~nm}$ (Figure 2), and roughly spherical shapes though other shapes are seen. The micrographs show that the particles tended to form larger aggregates, although most aggregates are believed to have formed during the air drying process prior to conducting the TEM imaging.

The measured values of the volume magnetic susceptibility of the $30 \mathrm{~nm}$ hematite nanoparticles themselves at room temperature, without any dispersant, was positive. This meant that the nanoparticles themselves did not exhibit diamagnetic behavior. The volume magnetic susceptibility using the Bartington MS2E probe low field sensor on the bulk $30 \mathrm{~nm}$ hematite material was about $1 \times$ $10^{-5}$ SI. The more accurate independent volume magnetic susceptibility on a 10 $\mathrm{ml}$ sample of the $30 \mathrm{~nm}$ hematite using the Bartington MS2B low field sensor was $0.5 \times 10^{-5} \mathrm{SI}$. Using the mass of the sample the mass magnetic susceptibility was calculated to be $21 \times 10^{-8} \mathrm{~m}^{3} \cdot \mathrm{kg}^{-1}$. 


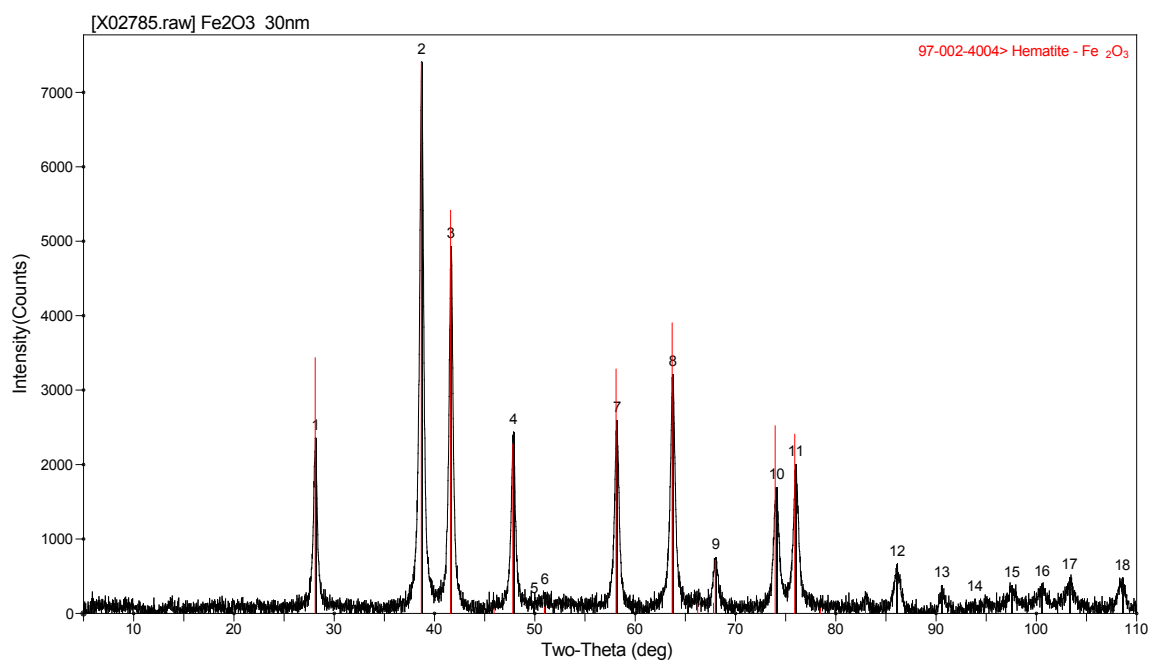

Figure 1. X-ray diffraction spectra of the spherical $30 \mathrm{~nm}$ hematite particles. The black line trace shows the observed spectra and the red vertical lines indicate the expected peaks for hematite.

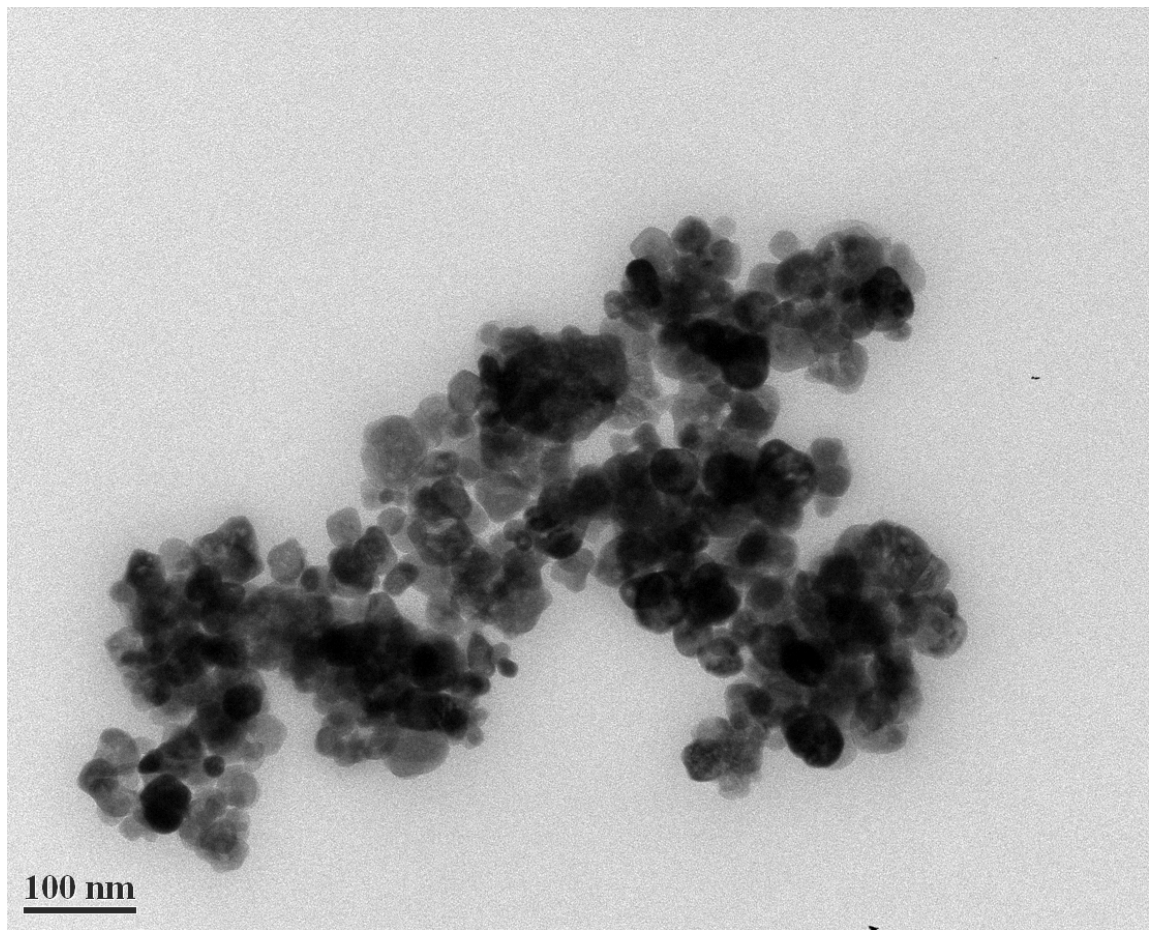

Figure 2. TEM photomicrograph of the $30 \mathrm{~nm}$ hematite particles. The scale bar is 100 $\mathrm{nm}$. The darker areas in the image are merely caused by some nanoparticles overlying one another.

Figure 3 shows the magnetic hysteresis curve of the $30 \mathrm{~nm}$ hematite nanoparticles (dispersed in calcium fluoride) from the VFTB measurements at room temperature. The curve shows a positive magnetization for positive magnetic field strengths, and is thus consistent with the two independent positive values of low field magnetic susceptibility determined from the Bartington sensor measurements. The magnetic hysteresis curve does not indicate diamagnetic 


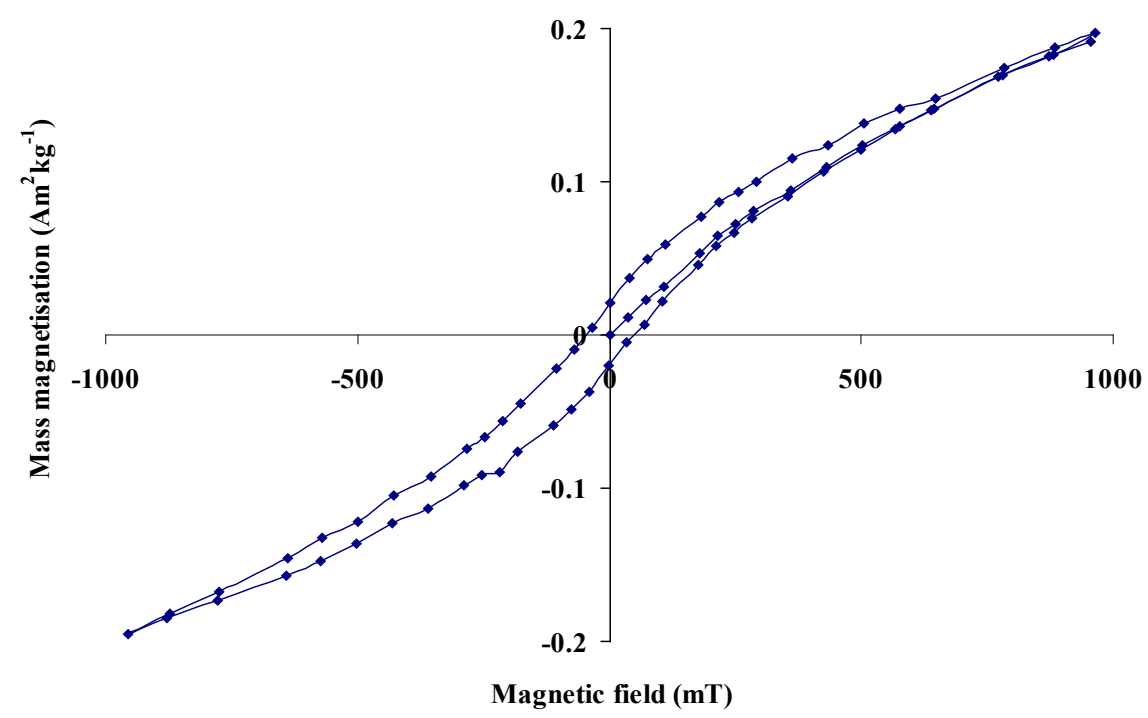

Figure 3. Magnetic hysteresis loop of the $30 \mathrm{~nm}$ hematite particles dispersed in calcium fluoride.

behavior of the hematite nanoparticles, but is consistent with hematite behaving as a canted antiferromagnetic material at room temperature.

For the dilutely dispersed hematite nanoparticles, however, the magnetic hysteresis curves were dominated by the dispersing agent (the diamagnetic water). Figure 4 shows the curve for the water alone. It is a pure diamagnetic signal comprising a straight line of negative slope passing through the origin. Figure 5 shows the magnetic hysteresis curve of the $40 \mathrm{~nm}$ hematite nanoparticles dilutely dispersed in the water. Whilst the diamagnetic signal of the water dominates at high applied field strength, a small kink in the hysteresis curve of Figure 5 is observed at low applied field and is indicative of the hematite nanoparticles. The slope of the magnetic hysteresis curve is positive at relatively low positive applied fields up to about $70 \mathrm{mT}$, and this is due to the $40 \mathrm{~nm}$ hematite particles. Figure 5 therefore demonstrates that a very dilutely dispersed sample of hematite nanoparticles can at first glance give the appearance of diamagnetic behavior, but the diamagnetic features (at applied fields greater than about $70 \mathrm{mT}$ in this case) are due entirely to the diamagnetic dispersing agent (water in this case). The magnetic hysteresis curve for the $8 \mathrm{~nm}$ hematite nanoparticles dilutely dispersed in the water was identical to that of Figure 4 and therefore completely dominated by the diamagnetic signal of the water. The reason we observed no small kink at low fields in this case could be partly because the concentration was lower at $140 \mathrm{mg} / \mathrm{L}$ for the $8 \mathrm{~nm}$ hematite nanoparticle dispersion compared to 160 $\mathrm{mg} / \mathrm{L}$ for the $40 \mathrm{~nm}$ hematite nanoparticle dispersion. Also the intrinsic magnetic susceptibility of the $8 \mathrm{~nm}$ nanoparticles could be lower than for the $40 \mathrm{~nm}$ nanoparticles.

Our results suggest that the diamagnetic behavior reported in [1] is the result of their hematite nanoparticles being dilutely dispersed in a diamagnetic dispersant. Their paper gives no details of their dispersant, but it may be relevant to 


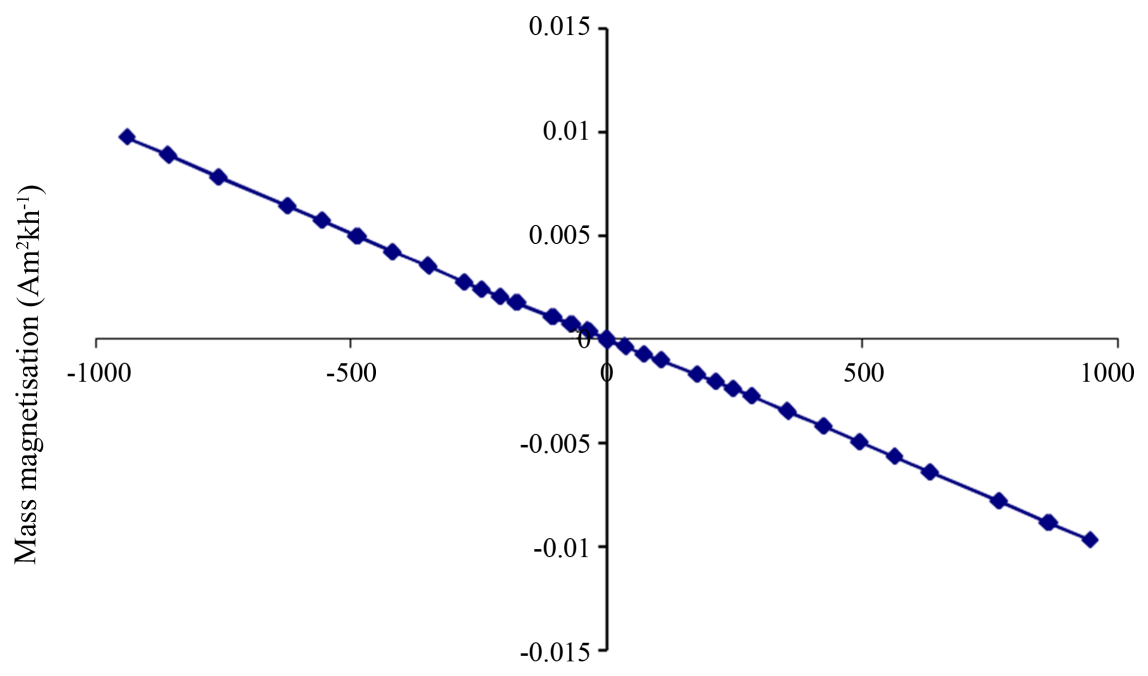

Magnetic field (mT)

Figure 4. Magnetic hysteresis curve of the diamagnetic water that was used to dilutely disperse the $8 \mathrm{~nm}$ and $40 \mathrm{~nm}$ hematite nanoparticles.

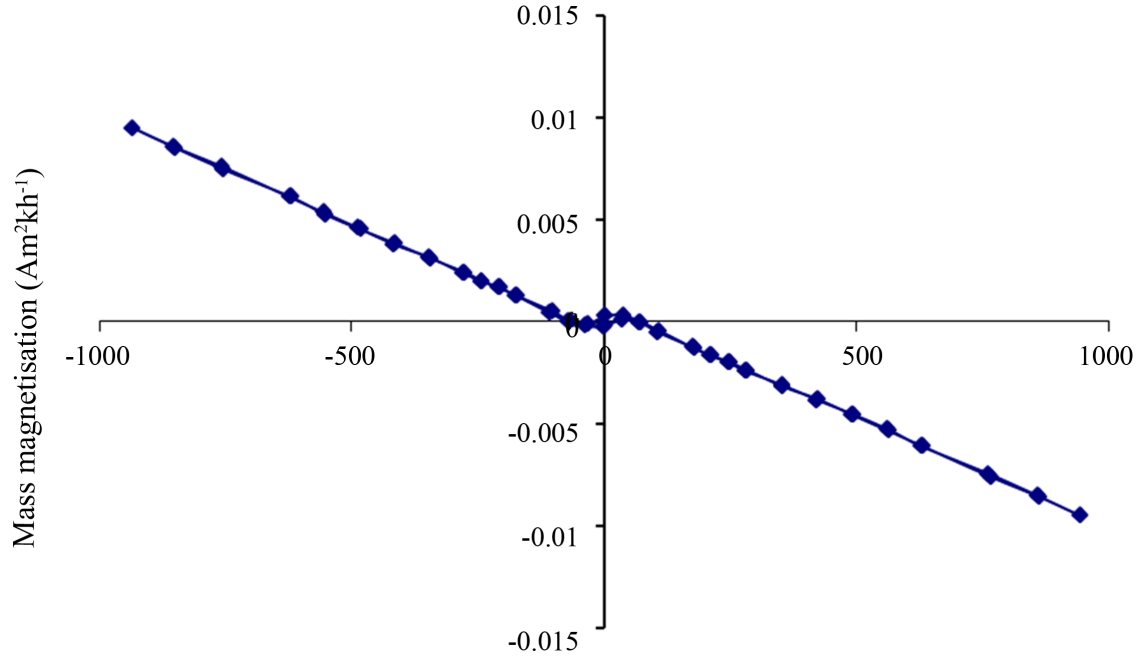

Magnetic field (mT)

Figure 5. Magnetic hysteresis curve of the $40 \mathrm{~nm}$ hematite nanoparticles dilutely dispersed in water. Note the "kink" in the curve at low applied magnetic fields, which is due to the hematite nanoparticles (and which is not seen in the pure water signal of Figure 4).

note that their sample which showed the "diamagnetic" behavior was the sample that was produced from the lowest concentration $(0.01 \mathrm{~mol} / \mathrm{L})$ of ferric nitrate $\left(\mathrm{Fe}\left(\mathrm{NO}_{3}\right)_{3}\right)$.

\section{Conclusions}

Our results suggest that the "diamagnetic" behavior reported by [1] in spindle-type hematite nanoparticles is very likely due to a diamagnetic dispersant dominating the magnetic properties, rather than any intrinsic diamagnetic behavior of the hematite nanoparticles themselves. For our $40 \mathrm{~nm}$ hematite nano- 
particles dilutely dispersed in water we demonstrated that the high field magnetic hysteresis curve was dominated by the diamagnetic property of the water, whilst the small low field kink in the curve (indicating positive magnetic susceptibility) was due to the hematite nanoparticles and consistent with the accepted view of hematite being a canted antiferromagnetic material at room temperature. The hysteresis curve of the $8 \mathrm{~nm}$ hematite nanoparticles (that were even more dilutely dispersed in water) was entirely dominated by the diamagnetic signal of the water. For the higher concentration samples containing $30 \mathrm{~nm}$ hematite nanoparticles, our observations of positive magnetic susceptibility, together with the magnetic hysteresis behavior, were also consistent with hematite being a canted antiferromagnetic material at room temperature.

\section{Acknowledgements}

We thank Particular $\mathrm{GmbH}$ for the dilutely dispersed hematite nanoparticles in water.

\section{References}

[1] Mi, Y., Zhang, X., Luo, J., Zhou, S., Zhang, H., Cheng, J. and Liu, F. (2006) Hydrothermal Fabrication of Spindle-Type $\alpha-\mathrm{Fe}_{2} \mathrm{O}_{3}$ Nanoparticle and Its Magnetic Property. Proceedings of the $1^{\text {st }}$ IEEE International Conference on Nano/Micro Engineered and Molecular Systems, Zhuhai, 18-21 January 2006, 519-522.

[2] Dunlop, D.J. and Özdemir, Ö. (1997) Rock Magnetism Fundamentals and Frontiers. Cambridge University Press, Cambridge. https://doi.org/10.1017/CBO9780511612794

[3] Özdemir, Ö., Dunlop, D.J. and Berquó, T.S. (2008) Morin Transition in Hematite: Size Dependence and Thermal Hysteresis. Geochemistry, Geophysics, Geosystems, 9, 1-12. https://doi.org/10.1029/2008GC002110

\section{Scientific Research Publishing}

Submit or recommend next manuscript to SCIRP and we will provide best service for you:

Accepting pre-submission inquiries through Email, Facebook, LinkedIn, Twitter, etc. A wide selection of journals (inclusive of 9 subjects, more than 200 journals)

Providing 24-hour high-quality service

User-friendly online submission system

Fair and swift peer-review system

Efficient typesetting and proofreading procedure

Display of the result of downloads and visits, as well as the number of cited articles

Maximum dissemination of your research work

Submit your manuscript at: http://papersubmission.scirp.org/

Or contact jmp@scirp.org 